\title{
Solute transport in fractured media - analysis of non-reversibility in tracer tests
}

\author{
N. E. V. Rodrigues ${ }^{1}$, J. L. M. P. de Lima ${ }^{2}$, and F. F. Cruz ${ }^{3}$ \\ ${ }^{1}$ Earth Sciences Department, Faculdade de Ciências e Tecnologia da Universidade de Coimbra, Largo Marquês de Pombal, \\ 3000-272 Coimbra, Portugal \\ ${ }^{2}$ Civil Engineering Department, Faculdade de Ciências e Tecnologia da Universidade de Coimbra, Rua Luís Reis Santos - \\ Pólo II Universidade de Coimbra, 3030-788 Coimbra, Portugal \\ ${ }^{3}$ Civil Engineering Department, Escola Superior de Tecnologia e Gestão de Leiria, Morro do Lena - Alto do Vieiro, 2411-901 \\ Leiria, Portugal
}

Received: 11 December 2007 - Revised: 3 September 2008 - Accepted: 3 September 2008 - Published: 23 October 2008

\begin{abstract}
This paper describes several small-scale (laboratory) experiments designed to simulate solute transport through fractured formations. A block of granite was broken to produce a fracture similar to those found in natural environments. Seven holes were drilled in the block to intersect the fracture. Later these holes functioned as either inlet or outlet points. All the possible combinations of pairs of inlet-outlet points were used to set up the tracer tests that provided the data analysed in this paper.

The results indicate that reverse tracer tests do not necessarily provide symmetric results. Under some circumstances, the non-reversibility might be used to detect differences in the morphology of the fracture. The results also indicate that it is possible to estimate reasonably well the volume available for the circulation of the fluid by using transport models that neglect diffusion.
\end{abstract}

\section{Introduction}

It is important to study solute transport in fractured formations because the fractures can create conduits which become fast flow paths for the circulation of fluids and/or dissolved species. Fractured formations represent a large portion of the solid Earth. For instance, in Portugal, they cover about two thirds of the surface of the country. Even those formations that are considered porous media are affected by faulting, and the distribution of flow is far from uniform. Applications for accurate tests can be found, for instance, in the following areas: oil fields (e.g. Zemel, 1995), hydrogeology (e.g. Bear et

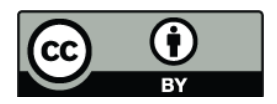

Correspondence to: N. E. V. Rodrigues (nelsonr@ci.uc.pt) al., 1993; Käss, 1998; Hill and Tiedman, 2007), radioactive waste disposal (e.g. Gylling et al., 1998). The simplifications that have been introduced give rise to inaccuracies that are not usually taken into account.

Fluid flow in fractured media has been analysed using either a porous media equivalent or a parallel plate model. The use of one or other approach depends on the scale of the problem, which in turn dictates whether a representative elementary volume or REV can be defined (e.g. Bear et al., 1993). The porous media equivalent is tackled by means of a generalized Darcy's law (e.g. Bear and Verruijt, 1987). The discrete case normally implies the use of the so-called cubic law that resulted, in particular, from the works of Snow (1969) and Louis (1974). Other studies introduced some tuning of the original formulas (a review can be found in Lee and Farmer, 1993; Cook, 2003). However the flow of fluids in fractured media follows a channelled pattern and current understanding of the process and related phenomena is still fraught with difficulties. Montazer (1987) and Rasmussen and Evans (1987) describe laboratory and field methods used in unsaturated fractured rock and the problems encountered. An example of a major experiment where a channel model approach has been used is described in Gylling et al. (1998). A comprehensive review of the state of the art in this field of knowledge is "Rock Fractures and Fluid Flow - Contemporary Understanding and Applications" (NRC, 1996). The importance of understanding fractures and associated phenomena is highlighted in the beginning of this work. Three specific questions are addressed there: (1) "How can fractures that are significant hydraulic conductors or barriers be identified, located and characterized", (2) "How do flow and transport occur in fracture systems?" and (3) "How can changes in fracture systems be predicted and controlled?". This paper

Published by Copernicus Publications on behalf of the European Geosciences Union and the American Geophysical Union. 

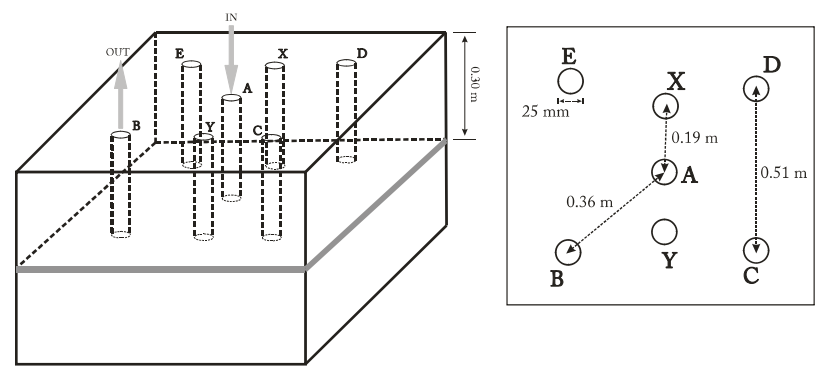

Fig. 1. Set-up of the laboratory experiments (adapted from Cruz, 2000).

fits into the context of the second question, focusing on solute transport. The propagation rate (Rodrigues, 1994) of solute transport is a fast process (e.g. Novakowski et al., 2004).

Current modelling of solute transport in fractured media is a two-step procedure: (1) resolution of the flow problem, and (2) resolution of the transport problem (e.g. Bear and Verruijt, 1987; Hill and Tiedman, 2007). The flow problem supplies medium flow velocity values for the transport problem. Analytical and numerical solutions exist for a number of cases (recent attempts can be found, for instance, in Chen et al., 2007; Novakowski and Bogan, 1999; Carlier et al., 2006; Coronado and Ramírez-Sabag, 2005). These methods can lead to wrong estimates because the distribution of velocities can create flow paths very different from those foreseen by the modelling (Rodrigues, 1994).

The initial aim of this work was the evaluation of procedures for the calculation of the volume available for the circulation of the fluids in fractured media. However the experiments raised difficulties like the non-uniqueness of answers for similar flow conditions, and so some of the questions and proposed explanations are shared in this paper.

In the laboratory, an artificial fracture in a granite block was intersected by several holes. Several tracer tests were then conducted. The tests intended to: (i) analyse the advection and dispersion of solutes in fractured media and (ii) assess the importance of the velocity field in the dispersion of solutes in fractured media.

\section{Experimental procedure and set-up}

One granite block was used for the laboratory tests. The block came from a quarry which is located about $100 \mathrm{~km}$ north-east of Coimbra (Portugal). The quarry site is dominated by late Hercynian granite (Geological Map of Portugal, Sheet 17-C, scale 1:50000). This rock is porphyritic granite, grey with no preferential orientation of the feldspathic phenocrysts. This is a light-coloured rock with $10-15 \%$ mafic minerals.

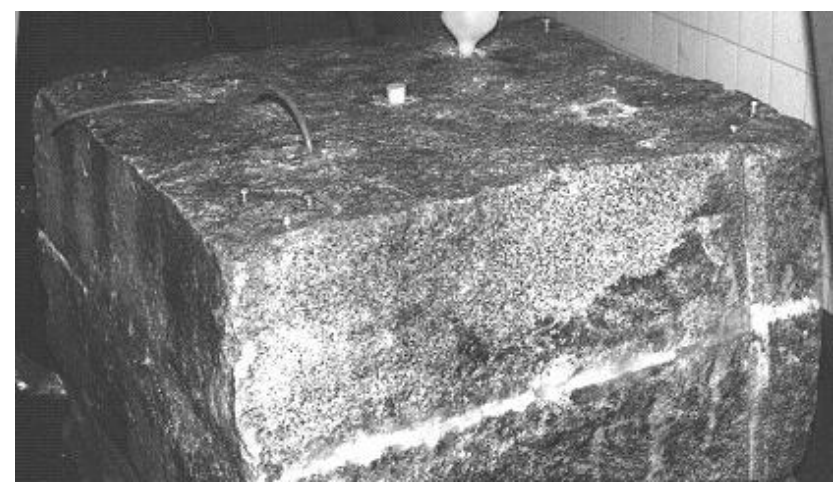

Fig. 2. Granite block and detail of the sealed fracture.

The block was split longitudinally in order to create an artificial fracture (Figs. 1 and 2) that was later used as the flow path of the water. The sides of the fracture were sealed with a silicone sealant, to guarantee that all the water circulated inside the block and to prevent possible leaks.

Tap water was used in the tests and some of its characteristics are given in Table 1.

The block of granite used in the laboratory tests measured approximately $1 \mathrm{~m} \times 1 \mathrm{~m} \times 0.60 \mathrm{~m}$. The artificial horizontal fracture was intersected by seven vertical holes, $0.025 \mathrm{~m}$ in diameter (Figs. 1 and 2).

During each test a constant flow of water was injected in one of the holes and extracted from another, while all the others were kept shut. An injection-extraction pair of wells was simulated in each case. The measured volume corresponding to two holes plus the fracture was 2.6 litres.

At the beginning of each test, small amounts of soluble chemicals were injected into the water (pulse injection) and from that moment on (initial time, $t_{0}$ ) the variation of the concentration of the solute in the outlet hole was registered.

The tests were organized in two groups: (i) using the combinations of five holes (A, B, C, D, and E, respectively, centre and vertices of a square) and (ii) a dipole with two holes (designated "X" and "Y"). With this framework it was possible to plan 22 (twenty-two) different configurations (A (in) to B (out), B to A, .., E to D, $\mathrm{X}$ to $\mathrm{Y}$ and $\mathrm{Y}$ to $\mathrm{X}$ ). As three chemical solutes were used in the tracer tests (sodium chloride, sulphorhodamine and uranine), a total of 66 (sixty-six) tracer tests were carried out. Table 2 summarizes the general conditions of the tests.

On average each test lasted one hour. It was observed that most of injected tracer left the system within thirty minutes. The temperature of the water and the $\mathrm{pH}$ remained constant for the duration of the tests (around $18^{\circ} \mathrm{C}$ and $\mathrm{pH}$ of 6.8).

The concentration of sodium chloride $(\mathrm{NaCl})$ was not measured directly. Instead the conductivity of the water was measured with a digital conductivity meter. The device used was able to store 200 readings that were later transferred to a flow-sheet for data processing. 
Table 1. Characteristics of the water used in the experiments (SMASC, 2001).

\begin{tabular}{cccccc}
\hline $\begin{array}{c}\text { Temperature } \\
\left({ }^{\circ} \mathrm{C}\right)\end{array}$ & $\begin{array}{c}\text { Conductivity } \\
\left(\mu \mathrm{S} \mathrm{cm}^{-1}\right)\end{array}$ & $\begin{array}{c}\mathrm{pH} \\
(-)\end{array}$ & $\begin{array}{c}\mathrm{Cl} \\
\left(\mathrm{mg} \mathrm{L}^{-1}\right)\end{array}$ & $\begin{array}{c}\mathrm{SO}_{4} \\
\left(\mathrm{mg} \mathrm{L}^{-1}\right)\end{array}$ & $\begin{array}{c}\mathrm{NH}_{4} \\
\left(\mathrm{mg} \mathrm{L}^{-1}\right)\end{array}$ \\
\hline 18 & 107 & 7.0 & 12.5 & 10.9 & 0.032 \\
\hline
\end{tabular}

\begin{tabular}{cccccccc}
\hline $\begin{array}{c}\mathrm{NO}_{3} \\
\left(\mathrm{mg} \mathrm{L}^{-1}\right)\end{array}$ & $\begin{array}{c}\mathrm{NO}_{2} \\
\left(\mathrm{mg} \mathrm{L}^{-1}\right)\end{array}$ & $\begin{array}{c}\mathrm{HCO}_{3} \\
\left(\mathrm{mgL}^{-1}\right)\end{array}$ & $\begin{array}{c}\mathrm{Na} \\
\left(\mathrm{mgL}^{-1}\right)\end{array}$ & $\begin{array}{c}\mathrm{Ca} \\
\left(\mathrm{mg} \mathrm{L}^{-1}\right)\end{array}$ & $\begin{array}{c}\mathrm{K} \\
\left(\mathrm{mg} \mathrm{L}^{-1}\right)\end{array}$ & $\begin{array}{c}\mathrm{Fe} \\
\left(\mu \mathrm{gL}^{-1}\right)\end{array}$ & $\begin{array}{c}\mathrm{Zn} \\
\left(\mu \mathrm{gL}^{-1}\right)\end{array}$ \\
\hline 3.8 & 0.003 & 27.6 & 10.3 & 7.9 & 1.3 & 70 & 140 \\
\hline
\end{tabular}

Table 2. General conditions of the tracer tests.

\begin{tabular}{lccc}
\hline Tracer & Concentration $(\mathrm{g} / \mathrm{L})$ & Amount injected $(\mathrm{ml})$ & Mean flow rate $(\mathrm{L} / \mathrm{s})$ \\
\hline Sodium chloride & 250 & 10 & 0.0167 \\
Sulphorhodamine & 0.0001 & 10 & 0.0167 \\
Uranine & 0.0001 & 10 & 0.0167 \\
\hline
\end{tabular}

The concentrations of sulphorhodamine and uranine (sodium fluorescein) were measured with a portable fluorimeter. This device recorded the measurements on a disk and they were later transferred to a computer. The software converts the measured fluorescence values into concentration values. The system was able to record two simultaneous tracer tests.

\section{Methodology}

The tests involved the continuous injection of water through the fractured block of granite. The flow rate and the temperature were kept constant during each tracer test.

It is useful to recall the notion of characteristic time, $t_{c}(s)$, the time necessary for some transport phenomena to propagate a certain distance $L(\mathrm{~m})$, which applies to diffusion type equations with the parameters adapted to each case (e.g. Rodrigues, 1994):

$t_{c}=\frac{L^{2}}{4 D}$

where $D\left(\mathrm{~m}^{2} / \mathrm{s}\right)$ is the diffusivity coefficient of the specific phenomena.

The rock matrix is, for practical purposes, impermeable in relation to fluid flow, taking into account the presence of a rather more permeable joint. For the tracer tests the diffusivity coefficient is a diffusivity of momentum and consequently it is given by $v L^{*}$ (where $\boldsymbol{V}$ is the fluid flow velocity $(\mathrm{m} / \mathrm{s}$ ) and $L^{*}$ is a characteristic length). In this case the characteristic time is of the order of a couple of minutes.

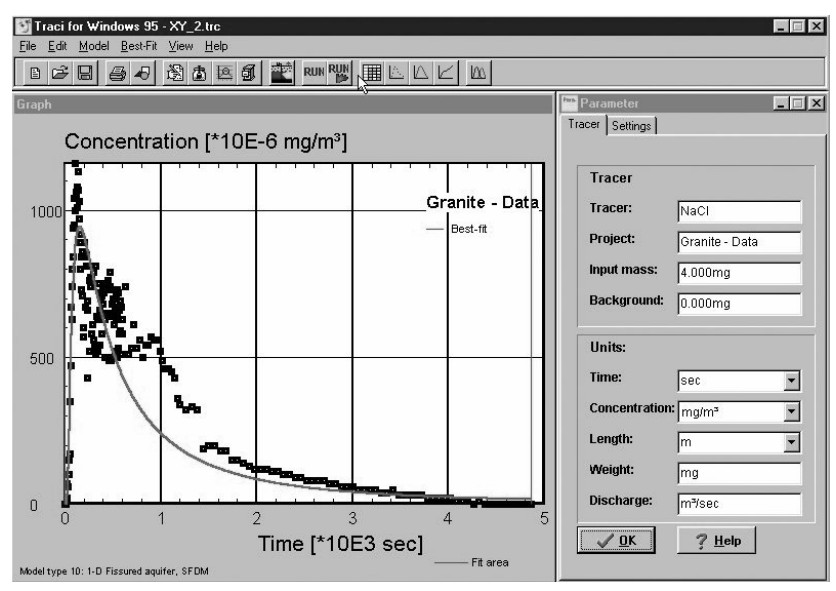

Fig. 3. Example of a tracer test ( $\mathrm{X}$ to $\mathrm{Y}$ ) and the best fit curve from Traci95.

By recording the concentration of the solutes (in the cases of uranine and sulphorhodamine) or the conductivity of the water (in the case of sodium chloride) it was possible to calculate the moments of the tracer test curves or the parameters of the "Single Fissure Dispersion Model" (SFDM) developed by Maloszewski and implemented in the software Traci95 (Käss, 1998). Figure 3 below shows the screen output of one of the interpreted results obtained with this software.

A summary of the results is given in the next section. The theoretical interpretation of the transport of the chemical solutes, which is given by the advection-dispersion equation, is 
outlined in Appendix A. Further details are available in the literature (e.g., Käss, 1998; Bear and Verruijt, 1987; Levenspiel, 1972; Cook, 2003).

In this study two different approaches have been used to estimate system parameters. One is probabilistic and it is based on the characterisation of the probability density functions of the residence times (e.g. Levenspiel, 1972). The second approach is deterministic. First a geometric model of the system is assumed. Then the flow problem followed by the transport problem are solved (e.g. Bear and Verruijt, 1987).

The residence time distribution function, $f(t) d t$, (Levenspiel, 1972) represents the probability that a particle picked at random has a time of residence inside the system between $t$ and $t+d t$. This can be expressed as:

$f(t) d t=\frac{C(t)}{\int_{0}^{\infty} C\left(t^{\prime}\right) d t^{\prime}} d t=\frac{Q}{M} C(t) d t$

where $f(t)$ is the density function of the residence time $\left(\mathrm{s}^{-1}\right) ; C(t)$ is the mass concentration measured at the exit $\left(\mathrm{kg} / \mathrm{m}^{3}\right) ; Q$ represents the flow rate $\left(\mathrm{m}^{3} / \mathrm{s}\right)$ and $M$ is the mass of tracer injected "instantaneously" (kg).

Recalling that in the tests with sodium chloride the electrical conductivity was measured instead of the concentration and knowing that the relation between conductivity and concentration is linear:

$C(t)=K C_{v}(t)$

where $C(t)$ is the sodium chloride mass concentration $\left(\mathrm{kg} / \mathrm{m}^{3}\right), C_{v}(t)$ is the conductivity $(\mathrm{S}), K$ is a proportionality constant $\left(\mathrm{kg} / \mathrm{m}^{3} / \mathrm{S}\right)$, and $t$ is the time (s).

Substituting in (2) the relationship (3) we obtain:

$$
f(t) d t=\frac{K C_{v}(t) d t}{\int_{0}^{\infty} K C_{v}\left(t^{\prime}\right) d t^{\prime}}=\frac{C_{v}(t) d t}{\int_{0}^{\infty} C_{v}\left(t^{\prime}\right) d t^{\prime}}
$$

It should be pointed out that the concentration and conductivity values used in the equations above are corrected values: in the concentration case the values do not take into account the initial background concentration, and in the conductivity case the values do not take into account the initial conductivity of the water. This means that:

$$
C_{v}(t)=C_{v}^{\text {measured }}(t)-C_{v}^{\text {water }}(t)
$$

In the data processing it was assumed that the conductivity of the water remained constant and that it was equal to the value read at the beginning of each test before the injection of the sodium chloride.

Finally for the numerical calculation of $f(t) d t$ a simple discretization has been used:

$$
f\left(t_{j}\right)=\frac{C_{v}(t)}{\int_{0}^{\infty} C_{v}\left(t^{\prime}\right) d t^{\prime}} \cong \frac{C_{v}\left(t_{j}\right)}{\sum_{i=1}^{n} C_{v}\left(t_{i}\right) \Delta t_{i}}
$$

with $\Delta t_{i}=t_{i}-t_{i-1}$ and $t_{0}=$ beginning of the test and $t_{n}=$ time at the end of the test.

$\bar{t}=\int_{0}^{\infty} t f(t) d t \cong \frac{\sum_{j=1}^{n} t_{j} C_{v}\left(t_{j}\right) \Delta t_{j}}{\sum_{i=1}^{n} C_{v}\left(t_{i}\right) \Delta t_{i}}$

where, $\bar{t}$ is the mean time and

$V_{f}=Q \bar{t}$

is the "volume of fluid circulation".

Dispersion parameters were not calculated using this approach because they would be moments of order two of the density function (variance or standard deviation) and they would not have an obvious physical meaning (i.e. they would not be directly related to physical characteristics of the flow).

Assuming a deterministic model, the data were processed with the software "Traci95" (Käss, 1998). It implements the SFDM model ("Single Fissure Dispersion Model - simulation of the transport through a fractured system with the possibility of diffusion into the solid matrix). This model was developed by Maloszewski (Käss, 1998). In the software the function $f(t)$ assumes the role of a normalized concentration function, resulting from an injection of a mass of tracer given by:

$M=\frac{1}{Q \int_{0}^{\infty} C(t) d t}$

where $Q$ is the flow rate $\left(\mathrm{m}^{3} / \mathrm{s}\right), M$ is the mass of the injected tracer $(\mathrm{kg})$.

The program allows the experimental data to be adjusted to the model. The adjustment parameters are: the mean time, the Peclet number and a parameter, $a^{1}$, which is defined as:

$a=\frac{n_{p} \sqrt{D_{p}}}{2 b}$

where $2 b$ is the fracture aperture $(\mathrm{m}), n_{p}$ is the porosity of the solid matrix $\left(\mathrm{m}^{3} / \mathrm{m}^{3}\right)$ and $D_{p}$ is the coefficient of diffusion $\left(\mathrm{m}^{2} / \mathrm{s}\right)$. This parameter describes the diffusion process for the solid.

As usual, the Peclet number is defined as $P e=\frac{L V}{D}$, where $L$ is some characteristic length, which, in the current context, represents the shortest distance between the injection and the extraction holes $(\mathrm{m}) ; \boldsymbol{V}$ is the average velocity of the flow $(\mathrm{m} / \mathrm{s})$; and $D$ is the coefficient of molecular diffusion $\left(\mathrm{m}^{2} / \mathrm{s}\right)$. High Peclet numbers represent solute transport dominated by advection, while low Peclet numbers correspond to transport where molecular diffusion dominates.

\footnotetext{
${ }^{1}$ Symbols $a, b$ and $n_{p}$ of this equation correspond to the nomenclature used in the software and they are used differently in this paper (e.g. $a$ is used for fracture aperture).
} 
Table 3. Tracer tests in the dipole $\mathrm{X}$ and $\mathrm{Y}$.

\begin{tabular}{|c|c|c|c|}
\hline & \multicolumn{3}{|c|}{ Sodium Chloride } \\
\hline & $\mathrm{X} \rightarrow \mathrm{Y}$ & $\mathrm{Y} \rightarrow \mathrm{X}$ & Delta \\
\hline Mean time $(s)^{*}$ & 1142 & 82 & 1.732 \\
\hline Mean time $(\mathrm{s})^{* *}$ & 994 & 251 & 1.194 \\
\hline Mean Volume $(1)^{*}$ & 17.8 & 1.2 & 1.747 \\
\hline Mean Volume (1)** & 15.5 & 3.7 & 1.229 \\
\hline Peclet Number & 0.6 & 8.8 & -1.745 \\
\hline
\end{tabular}

\begin{tabular}{lllllll}
\hline & \multicolumn{3}{c}{ Fluorescein } & \multicolumn{3}{c}{ Sulphorhodamine } \\
\hline & $\mathrm{X} \rightarrow \mathrm{Y}$ & $\mathrm{Y} \rightarrow \mathrm{X}$ & Delta & $\mathrm{X} \rightarrow \mathrm{Y}$ & $\mathrm{Y} \rightarrow \mathrm{X}$ & Delta \\
\hline Mean time (s) & 92 & 62 & 0.390 & 78 & 63 & 0.213 \\
Mean time (s)* & 292 & 286 & 0.021 & 250 & 218 & 0.137 \\
Mean Volume (1)* & 1.5 & 1.0 & 0.400 & 1.5 & 1.4 & 0.069 \\
Mean Volume (1) & 4.9 & 4.8 & 0.021 & 4.2 & 3.6 & 0.154 \\
Peclet Number & 18.2 & 19.2 & -0.053 & 12.2 & 1.6 & 1.536 \\
\hline
\end{tabular}

* - Values obtained from the output of Traci95.

** - Values calculated using Eqs. 7 (mean time) or 8 (mean volume).

\section{Results and discussion}

First it is convenient to visualize the general framework. What we have here is a set up made of a fracture plus a set of holes that provide the points into and out of the system. The volume available for the fluid flow is known. When a test is carried out two holes are open and all the others are shut so the volume is at the most 2.6 litres (fracture plus two holes). In chemical engineering this is also usually the case: the volume of the chemical reactor is known and so is the mean time. However this is not the usual case in geosciences: the volume of the system and the mean time are the main parameters being sought. So we were able to test the interpretations that are made with the models in current use and that have been described in the previous section.

The results for the dipole $\mathrm{X}$ and $\mathrm{Y}$ are summarized in Table 3 and Fig. 4 below. Table 3 shows the calculated parameters for the tracer testes that have used the holes " $X$ " and "Y". A parameter (Delta) has been devised for the purpose of comparing reverse tracer tests:

Delta $=[(\mathrm{H} 1 \rightarrow \mathrm{H} 2)-(\mathrm{H} 2 \rightarrow \mathrm{H} 1)] / 0.5 \times[(\mathrm{H} 1 \rightarrow \mathrm{H} 2)+(\mathrm{H} 2 \rightarrow \mathrm{H} 1)]$

where $\mathrm{H} 1 \rightarrow \mathrm{H} 2(\mathrm{H} 2 \rightarrow \mathrm{H} 1)$ represent the parameters calculated when the fluid flows from hole $\mathrm{H} 1$ to hole $\mathrm{H} 2$ (or from hole $\mathrm{H} 2$ to hole $\mathrm{H} 1$ ). The parameter Delta provides a measure of the deviation from symmetrical results (if the tests where symmetrical then Delta $=0$ ).

The most noticeable aspect of the results is the difference between the tests with sodium chloride and the tests with fluorescein or sulphorhodamine. Also remarkable is the difference between $(\mathrm{X} \rightarrow \mathrm{Y})$ tests and $(\mathrm{Y} \rightarrow \mathrm{X})$ tests, especially in

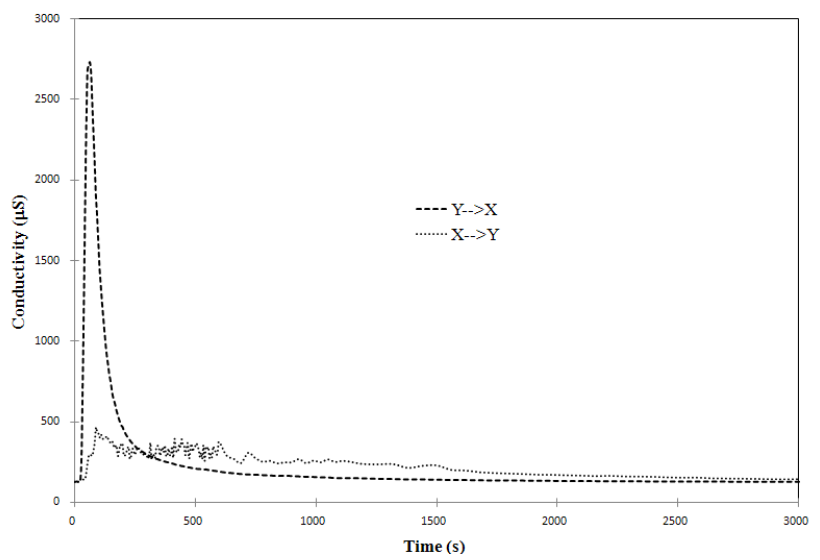

Fig. 4. Reverse tracer tests with noticeably different shapes.

the sodium chloride tests (Fig. 4). The most probable reason in both cases is the fact that the sodium chloride solution injected had a higher density than the water. As a result, if there are significant openings near the injection hole then the solution might sink there initially. It will be released later as it diffuses into the flowing water. If there are no pits near the injection point then the solution gets mixed with the flowing water more easily and so it travels faster through the system. The results show that there were some pits next to hole " $X$ ", acting as initial receivers of the heavier sodium chloride solution. Thus, one conclusion is that the transport might depend on the morphology of the areas around the injection holes, and the residence time of the sodium chloride molecules will be proportional to openings next to these areas. Therefore, the parameters calculated in the tests with sodium chloride do not correspond to the parameters of the flowing water.

For the remaining tests, the calculated results are synthesised in Fig. 5. In the figure there are pairs of values. The first value represents the volume, in litres, estimated from the parameters of the Traci95 simulation output and the second values (in brackets) represents the volume estimated after Eq. 8 (for instance, for the pair E (inlet) and D (outlet) the estimated volumes where 0.9 litres and 6.7 litres). It is quite clear that all flow paths are different, thus highlighting the non-reversibility of these processes.

Parameters characterizing the flow pattern without being affected by exogenous factors can be obtained from the tests with fluorescein or sulphorhodamine because they behave as inert tracers (e.g. Käss, 1998; Rodrigues, 1994). In addition to values from Tables 3, 4a and $\mathrm{b}$ give the calculated volumes for each pair of reverse tracer tests, using either the residence time theory (RTT, Eq. 8) or Traci95.

The differences observed between these two tracers (uranine and sulphorhodamine) are less significant and, as a first possibility, they could represent experimental fluctuations and/or observation errors. Therefore, it is relevant to analyse these results more thoroughly. First of all the differences, 


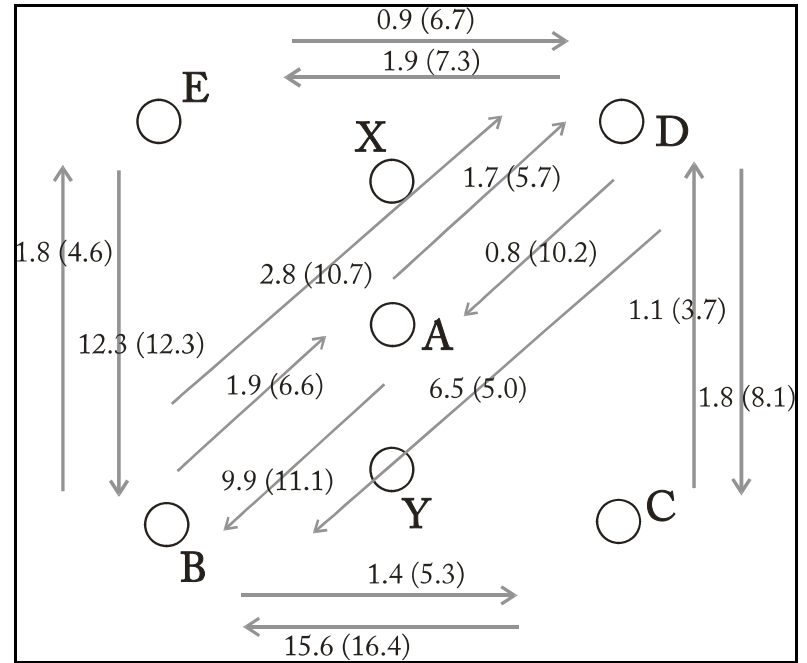

Fig. 5. Tests with $\mathrm{NaCl}$ - Volumes (1) from Traci95 and Eq. (8) (in brackets).

measured by the delta parameter, may be a order of magnitude different. Even the results from the mathematical model (used by Traci95), which are less affected by the tail of the tracer curve (unlike the RTT results), show consistent differences. This indicates that the flow paths in reverse tracer tests are not necessarily the same, and that the existing models are unable to simulate this non-reversibility. Comparing the shapes of the tracer curves it becomes clear that the differences are most significant in the tails of the reverse tracer tests. This fact is amplified if the data are plotted in a semilogarithmic graph. An example of tests using sulphorhodamine in holes A and B is shown in Fig. 6.

The volumes calculated in the tests (using Eq. 8) with fluorescein and sulphorhodamine varied between 1.7 and 4.9 litres. About eighty percent of the values, calculated using the residence time theory, were greater than 2.6 litres (the correct measured volume). This overvaluing of the calculated volumes is due, in principle, to the effects of molecular diffusion from areas of low flow, resulting in particles with very long residence times distorting the calculation of the mean residence times.

If we consider the results from Traci95, the calculated volumes vary from 0.6 to 2.9 litres, and only three of them are larger than 2.6 litres. These values are acceptable but it should be kept in mind that the model only explains the initial part of the curve, and it does not consider the values of the tail of the test. Thus in a situation where the volume is not known it is recommended to use Traci95 or some similar program, but keeping in mind that the value is a lower limit because it represents the portion of the total volume open to fluid flow where most of the fluid circulates.

The calculated values using the residence time should be regarded as upper limits and they should be analysed taking into account the possible existence of "dead ends".

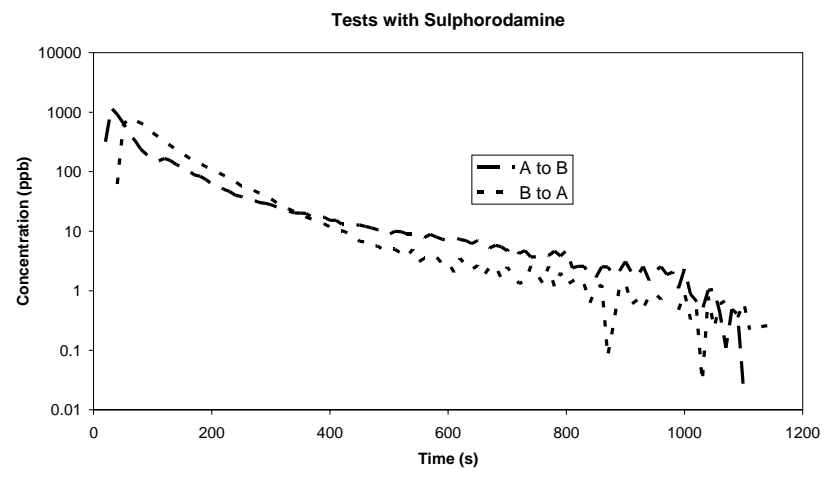

Fig. 6. Reverse tracer tests in holes A and B.

To sum up, the tests with fluorescein and sulphorhodamine confirm that these dye products are inert during the transport. Even though the results with sodium chloride were influenced by the density of the ejected fluid, they provided a positive side effect: they can be used to amplify the differences in the circulation between pairs of wells and thus give clues about the morphology of the fractures in the vicinity of the injection wells.

\section{Conclusions}

These experimental results allow the following conclusions to be drawn:

1. For the tests in the laboratory, the fluorescein and the sulphorhodamine were revealed to be good tracers, and no significant differences were found in the results. As expected, the observed transport was advection (high Peclet numbers). In fast tests, like those performed in the laboratory, the two tracers do not indicate heterogeneities in the surface of the fractures. Having said that, different results from the tests in pairs of holes have been obtained and the differences are clearer in the tails of the curves.

2. The tests with sodium chloride revealed an interesting side effect: the use of $\mathrm{NaCl}$ as a trace can be used to amplify morphological differences in flow paths. The "pulse" of injected sodium chloride might get caught in the bottom of the injection holes and their vicinity due to the higher density. It will be delayed in relation to the flowing water and this will give some indication about the heterogeneities of the fracture surface.

3. When the volume of the circulating fluid is not known (the usual case in geosciences) it is better to use a model/program like Traci95. However, the calculated value will be a lower limit, because it expresses the volume through which most of the fluid circulates, 
Table 4a. Calculated volumes for the tests with sodium fluorescein.

\begin{tabular}{lccrccr}
\hline & \multicolumn{2}{c}{ Volumes using RTT } & Delta & \multicolumn{2}{c}{ Volumes from Traci95 } & Delta \\
& Direct $\left(^{*}\right)$ & Reverse $\left(^{*}\right)$ & & Direct( $\left(^{*}\right)$ & Reverse $\left(^{*}\right)$ & \\
\hline $\mathrm{A} \Leftrightarrow \mathrm{B}$ & 2.7 & 2.5 & 0.077 & 0.7 & 1.1 & -0.444 \\
$\mathrm{~A} \Leftrightarrow \mathrm{D}$ & 2.9 & 4.2 & -1.115 & 1.6 & 1.2 & 0.286 \\
$\mathrm{~B} \Leftrightarrow \mathrm{D}$ & 3.8 & 4.3 & -0.123 & 1.9 & 2.2 & -0.146 \\
$\mathrm{~A} \Leftrightarrow \mathrm{C}$ & 2.1 & 2.3 & -0.091 & 1 & 1.1 & -0.095 \\
$\mathrm{~A} \Leftrightarrow \mathrm{E}$ & 3.6 & 3.6 & 0.000 & 0.8 & 0.8 & 0.000 \\
$\mathrm{C} \Leftrightarrow \mathrm{E}$ & 4.6 & 3.5 & 0.272 & 1.8 & 1.6 & 0.118 \\
$\mathrm{~B} \Leftrightarrow \mathrm{C}$ & 4.0 & 4.2 & -0.049 & 1.4 & 1.3 & 0.074 \\
$\mathrm{C} \Leftrightarrow \mathrm{D}$ & 4.6 & 4.0 & 0.140 & 1.1 & 1.1 & 0.000 \\
\hline
\end{tabular}

$\left(^{*}\right)$ - Direct meaning first hole to second hole and Reverse means second hole to first hole; for instance, for $\mathrm{A} \Leftrightarrow \mathrm{B}$ Direct means injection in A and extraction of the fluid in B.

Table 4b. Calculated volumes for the tests with sulphorhodamine.

\begin{tabular}{lccrccr}
\hline & \multicolumn{2}{c}{ Volumes using RTT } & Delta & \multicolumn{2}{c}{ Volumes from Traci95 } & Delta \\
& Direct $\left(^{*}\right)$ & Reverse $\left(^{*}\right)$ & & Direct $\left(^{*}\right)$ & Reverse $\left(^{*}\right)$ & \\
\hline $\mathrm{A} \Leftrightarrow \mathrm{B}$ & 2 & 2.2 & -0.095 & 0.6 & 1.4 & -0.800 \\
$\mathrm{~A} \Leftrightarrow \mathrm{D}$ & 2.8 & 3.6 & -0.250 & 1.6 & 1.6 & 0.000 \\
$\mathrm{~B} \Leftrightarrow \mathrm{D}$ & 3.9 & 4.2 & -0.074 & 2.7 & 2.7 & 0.000 \\
$\mathrm{~A} \Leftrightarrow \mathrm{C}$ & 1.7 & 2.3 & -0.300 & 0.9 & 1.1 & -0.200 \\
$\mathrm{~A} \Leftrightarrow \mathrm{E}$ & 2.9 & 3.4 & -0.159 & 1 & 0.9 & 0.105 \\
$\mathrm{C} \Leftrightarrow \mathrm{E}$ & 4.4 & 3.1 & 0.347 & 2.9 & 1.4 & 0.698 \\
$\mathrm{~B} \Leftrightarrow \mathrm{C}$ & 3.4 & 3.1 & 0.092 & 1.8 & 1.6 & 0.118 \\
$\mathrm{C} \Leftrightarrow \mathrm{D}$ & 3.2 & 3.6 & -0.118 & 1 & 1.3 & -0.261 \\
$\mathrm{D} \Leftrightarrow \mathrm{E}$ & 4.4 & 3.8 & 0.146 & 1 & 0.9 & 0.105 \\
\hline
\end{tabular}

$\left(^{*}\right)$ - Direct meaning first hole to second hole and Reverse means second hole to first hole; for instance, for A $\Leftrightarrow \mathrm{B}$ Direct means injection in $\mathrm{A}$ and extraction of the fluid in $\mathrm{B}$.

disregarding places where there is slow fluid circulation. The use of models based on residence time theory will give upper limits.

4. For the set up that has been used here it was expected that reverse tracers tests would provide similar results. However, reverse tracer tests can differ from one another quite a lot. The existing models cannot explain all the relevant features of the fluid flow.

\section{Appendix A}

\section{Solute transport and the advection-dispersion equation}

The advection-dispersion equation has been used to describe the transport of material in solution at a microscopic level (e.g. Bear and Verruijt, 1987):

$\frac{\partial c}{\partial t}=-\nabla \cdot(c \mathbf{V}-\mathbf{D} \nabla c)+\rho \mathbf{\Gamma}$ where $C(t)$ is the mass concentration, $\mathbf{V}$ is the velocity of the fluid (vector), $\mathbf{D}$ is the coefficient of molecular diffusion (tensor), $\rho$ is the density of the fluid and $\Gamma$ is the rate at which the mass of material is added to (or removed from) the fluid.

This equation states that the concentration of solute at a point varies as a result of (i) transport by the fluid $(C(t) v)$ (a linear process) and (ii) differences in concentration between points $(\mathbf{D} . \nabla C(t))$, a non-linear process). The last term $(\rho \Gamma)$ denotes the change in concentration due to chemical reactions or decay phenomena.

Analytical solutions exist only for a limited number of simplified cases (e.g. Käss, 1998) Most of the solutions are for the one-dimensional (1-D) flow with no chemical reaction. In this case the equation reduces to:

$\frac{\partial C}{\partial t}=D \frac{\partial^{2} C}{\partial x^{2}}-V \frac{\partial C}{\partial x}$

where $x$ represents the distance along the direction of the flow. 


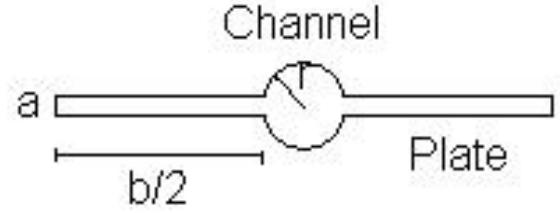

Fig. A1. Channel with wings (Rodrigues, 1994).

The complex geometry of porous media prevents the use of these equations. The definition of averaged variables (velocity and concentration) over a small portion of the porous media ("representative elementary volume" - REV) is one of the methods proposed to break this impasse (Bear and Verruijt, 1987). The total flux of solute through a porous medium is expressed as (Bear and Verruijt, 1987):

$q_{c, \text { total }}=\omega\left(C \mathbf{V}-\mathbf{D}_{h} \cdot \nabla C\right)$

where $q_{c \text {,total }}$ is the total flux of solute by advection, dispersion and diffusion, $\omega$ is the kinematic porosity, $C$ is the average concentration, $\boldsymbol{V}$ is the pore water velocity, $D_{h}=D+D^{*}$ is the coefficient of hydrodynamic dispersion (second order rank symmetrical tensor), $D$ is the coefficient of molecular diffusion in a porous medium (second order rank tensor), $D^{*}$ is the coefficient of mechanical dispersion (second order rank tensor).

The main effect of the averaging process is the introduction of an additional dispersive term. Three mechanisms cause this dispersion: (i) velocity variations across the pores; (ii) velocity variations between pores; (iii) differences in streamline path length.

For a macroscopic balance of solute transport, Eq. (A2) becomes (Bear and Verruijt, 1987):

$$
\frac{\partial \omega C}{\partial t}=-\nabla\left(C \mathbf{u}-\omega \mathbf{D} \cdot \nabla C-\omega \mathbf{D}^{*} \cdot \nabla C\right)-f+\omega \rho \Gamma-P C+R C_{r}
$$

where $\omega C$ is the mass of solute per unit volume of porous medium, $u$ is the seepage or Darcy's velocity, $f$ is the quantity of solute that leaves the fluid as a result of ion exchange and adsorption, $P$ is the pumping rate flow (per unit volume of porous medium per unit time), $R$ is the recharge rate flow (per unit volume of porous medium per unit time) and $C_{r}$ is the concentration of added fluid. Other terms have the same meaning as before.

With no interaction with the solid matrix, no decay and no pumping or recharge of fluid, Eq. (A4) simplifies to:

$$
\frac{\partial C}{\partial t}=\nabla\left(\mathbf{D}_{h} \cdot \nabla C-v C\right)
$$

where $V=u / \omega$ is the pore water velocity, $C=$ concentration of solute in the fluid and $D_{h}=$ coefficient of hydrodynamic dispersion.
Equation (A5) is the one normally adopted in studies of solute transport through porous media. In the case of fractured media there is a combination of several effects; the most important of which is the fact that the fluid flow is controlled by channelling. This means that most of the flow occurs in a number of dendritic pathways in the fracture surface, with average apertures greater than the average aperture of the fracture. The volume of the channels represents a small fraction of the total volume open to flow. A simplified model can be used to show that most of the fluid flows through a small percentage of the volume available for the flow. The model is a combination of a parallel plate with a pipe, referred to below as a "channel with wings" (Fig. A1, adapted from Rodrigues, 1994).

The formulas, that describe the fluid flow between smooth parallel plates and in smooth pipes assuming Poiseuille flow, are used in the calculations below.

The distribution of flow mentioned (10\% of the fracture's volume open to fluid flow is swept along by more than $80 \%$ of the fluid) is used to constrain the volumes of the plate and of the pipe. The volume of the pipe is assumed to be 10 times smaller than the volume of the plate. In the calculations the radius of pipe, $r$, is 10 times the aperture of the plate, $a$ (assume $100 \mu \mathrm{m}$ and $10 \mu \mathrm{m}$, respectively).

The calculated velocity of the fluid flowing through the pipe is 150 times larger than the velocity of the fluid caught in the plate for the same hydraulic gradient and viscosity of the fluid:

$\frac{u_{\text {pipe }}}{u_{\text {plate }}}=150$

where $u$ represents the velocity of the fluid in the pipe or in the plate.

So a particle of tracer travelling in the plate is, on average, 150 times slower than one circulating through the pipe. The ratio between flow rates is:

$\frac{Q_{\text {pipe }}}{Q_{\text {plate }}}=15$

i.e. through a volume which is 10 times smaller flows 15 times more fluid.

The value of the breadth of the plate is $b \cong 3 \mathrm{~cm}$ or $b / 2=1.5 \mathrm{~cm}$ each side of the pipe, which is a physically acceptable figure.

The volume associated with regions of slow-moving fluid ("the wings") can be an order of magnitude larger than the volume of the "channels" but most of the fluid flows through the "channels" even though they have a much smaller volume than the plates.

Acknowledgements. Fundação para a Ciência e Tecnologia (Portuguese Foundation for Science and Technology) provided financial support through the Project PTDC/ECM/70456/2006.

The tests were conducted in the Laboratory of Hydraulics, Water Resources and Environment of the Civil Engineering Department 
of the University of Coimbra. The support from the Department of Earth Sciences of the University of Coimbra and IMAR - Institute of Marine Research is also acknowledged.

The comments of the anonymous reviewers helped to improve significantly this paper. Their effort is commended.

Edited by: A. Tarquis

Reviewed by: four anonymous referees

\section{References}

Bear, J. and Verruijt, A.: Modeling Groundwater Flow and Pollution, D. Reidel Pub. Comp., Dordrecht, The Netherlands, 1987.

Bear, J., Tsang, C. F., and de Marsily, G.: Flow and Contaminant Transport in Fractured Rock, Academic Press, New York, 1993.

Carlier, E., El Khattabi, J., and Potdevin, J. L.: Solute transport in sand and chalk: a probabilistic approach, Hydrological Processes, John Wiley \& Sons, 20, 1047-1055, 2006.

Chen, J. S., Chen, C. S., and Chen, C. Y.: Analysis of solute transport in a divergent flow tracer test with scale-dependent dispersion, Hydrological Processes, John Wiley \& Sons, 21, 25262536, 2007.

Cook, P. G.: A guide to regional groundwater flow in fractured rock aquifers, CSIRO, Australia, 2003.

Coronado, M. and Ramírez-Sabag, J.: A New Analytical Formulation for Interwell Finite-Step Tracer Injection Tests in Reservoirs, Transport in Porous Media, Springer, 60, 339-351, 2005.

Cruz, F. F.: Dispersão de Contaminantes em Meios Fracturados - Ensaios Experimentais com Traçadores. (Contaminant dispersion in fractured media - Experimental tests with tracers), M. Sc. Thesis, Faculty of Science and Technology of the Coimbra University, Portugal, 2000 (in Portuguese).

Gylling, B., Birgersson, L., Moreno, L., and Neretnieks, I.: Analysis of a long-term pumping and tracer test using the channel network model, J. Contam. Hydrol., 32, 203-222, 1998.

Hill M. C. and Tiedman C. R.: Effective Groundwater Model Calibration, John Wiley, 2007.

Käss, W.: Tracing Technique in Geohydrology, Taylor \& Francis Ltd, Rotterdam, The Netherlands, 1998.
Lee, C.-H. and Farmer, I.: Fluid Flow in Discontinuous Rocks, Chapman \& Hall, London, UK, 1993.

Levenspiel O.: Chemical Reaction Engineering, 2nd edition, John Wiley \& Sons., New York, USA, 1972.

Louis, C.: Introduction à l'hydraulique des roches (Introduction to rock hydraulics). Bulletin Bureau de Recherches Géologiques et Minières 1974; III-4: 283-356, 1974.

Montazer, P.: Monitoring hydrologic conditions in the vadose zone in fractured rocks, Yucca Mountain, Nevada, in: Flow and Transport through Unsaturated Fractured Rock, edited by: Evans, D. D. and Nicholson, T. J., Geophys. Monogr. Ser., Vol. 42, 1st ed., AGU, Washington, D. C., 31-42, 1987.

Novakowski, K. S. and Bogan, J. D. A.: Semi-analytical model for the simulation of solute transport in a network of fractures having random orientations, International Journal for Numerical and Analytical Methods in Geomechanics, John Wiley \& Sons, 23, 317-333, 1999.

Novakowski, K. S., Bickerton, G., and Lapcevic, P.: Interpretation of injection-withdrawal tracer experiments conducted between two wells in a large single fracture, Jo. Contam. Hydrol., Elsevier, 73, 227-247, 2004.

NRC: Committee on Fracture Characterization and Fluid Flow, National Research Council, Rock Fractures and Fluid Flow - Contemporary Understanding and Applications, National Academy Press, Washington, D.C., USA, 1996.

Rasmussen, T. C. and Evans D. D.: Unsaturated flow and transport through fractured rock related to high-level waste repositories. Tech. Rep. NUREG/CR-4655, US Nucl. Reg. Comm., Washington, D.C., 1987.

Rodrigues, N.: The Interpretation of Tracer Curves in Hot Dry Rock Geothermal Reservoirs. Ph.D. Thesis, Exeter University, UK, 1994.

SMASC: Boletim periódico n. ${ }^{\circ}$ S17/01, Laboratório Controlo Qualidade dos Serviços Municipalizados de Água e Saneamento de Coimbra (SMASC), Coimbra, Portugal, 2001 (in Portuguese).

Snow, D. T.: Anisotropic permeability of fractured media, Water Resour. Res., 5(6), 1273-1289, 1969.

Zemel, B.: Tracers in the oil field, Elsevier, Amsterdam, The Netherlands, 1995. 